\title{
GM130 regulates pulmonary surfactant protein secretion in alveolar type II cells
}

\author{
Qianqian Pang ${ }^{1,3}$, Chunyi Liu ${ }^{1}$, Yulong Qiao ${ }^{4}$, Jian Zhao ${ }^{4}$, Sin Man Lam ${ }^{1}$, Mei Mei ${ }^{1}$, \\ Guanghou Shui ${ }^{1}$, Shilai Bao ${ }^{1,2^{*}} \&$ Qiuling $\mathrm{Li}^{4^{*}}$ \\ ${ }^{1}$ State Key Laboratory of Molecular Developmental Biology, Institute of Genetics and Developmental Biology, The Innovative Academy of Seed \\ Design, Chinese Academy of Sciences, Beijing 100101, China; \\ ${ }^{2}$ School of Life Sciences, University of Chinese Academy of Sciences, Beijing 100049, China; \\ ${ }^{3}$ Institute of Forensic Medicine and Laboratory Medicine, Jining Medical University, Jining 272067, China; \\ ${ }^{4}$ Department of Health Sciences, Institute of Physical Science and Information Technology, Anhui University, Hefei 230601, China
}

Received September 22, 2020; accepted January 5, 2021; published online March 16, 2021

\begin{abstract}
Pulmonary surfactant is a lipid-protein complex secreted by alveolar type II epithelial cells and is essential for the maintenance of the delicate structure of mammalian alveoli to promote efficient gas exchange across the air-liquid barrier. The Golgi apparatus plays an important role in pulmonary surfactant modification and secretory trafficking. However, the physiological function of the Golgi apparatus in the transport of pulmonary surfactants is unclear. In the present study, deletion of GM130, which encodes for a matrix protein of the cis-Golgi cisternae, was shown to induce the disruption of the Golgi structure leading to impaired secretion of lung surfactant proteins and lipids. Specifically, the results of in vitro and in vivo analysis indicated that the loss of GM130 resulted in trapping of Sftpa in the endoplasmic reticulum, Sftpb and Sftpc accumulation in the Golgi apparatus, and an increase in the compensatory secretion of Sftpd. Moreover, global and epithelial-specific GM130 knockout in mice resulted in an enlargement of alveolar airspace and an increase in alveolar epithelial autophagy; however, surfactant repletion partially rescued the enlarged airspace defects in GM130-deficient mice. Therefore, our results demonstrate that GM130 and the mammalian Golgi apparatus play a critical role in the control of surfactant protein secretion in pulmonary epithelial cells.
\end{abstract}

GM130, Golgi apparatus, surfactant protein, Alveolar type II cells

Citation: $\quad$ Pang, Q., Liu, C., Qiao, Y., Zhao, J., Lam, S.M., Mei, M., Shui, G., Bao, S., and Li, Q. (2022). GM130 regulates pulmonary surfactant protein secretion in alveolar type II cells. Sci China Life Sci 65, 193-205. https://doi.org/10.1007/s11427-020-1875-X

\section{INTRODUCTION}

The pulmonary system is the largest surface of our body that is exposed to the environment to facilitate its gas exchange function. The alveoli occupy more than $99 \%$ of the internal surface area of the lung and are closely connected with the surrounding capillaries to form an air-blood barrier, which directly participates in gas exchange (Branchfield et al., 2016; Morrisey and Hogan, 2010; Yu et al., 2007). The al-

*Corresponding authors (Shilai Bao, email: slbao@genetics.ac.cn; Qiuling Li, email: qlli@ahu.edu.cn) veolar surface is lined by two types of epithelial cells, alveolar type I (ATI) cells and alveolar type II (ATII) cells (Morrisey and Hogan, 2010). Flattened ATI cells cover approximately $95 \%$ of the alveolar surface serving as a thin barrier between the blood and air and functioning as the signaling partners of ATII cells (Andreeva et al., 2007). ATII cells are stem cells in the alveolar regions that can to selfrenew and differentiate into ATI cells (Wang et al., 2007); however, the main function of ATII cells is to synthesize and secrete pulmonary surfactant (Orgeig et al., 2010).

Pulmonary surfactant is a lipid-protein complex that forms 
interfacial films that line the alveolar airspace to reduce surface tension at the air-blood interface and protect the alveolar architecture during the respiration cycle (Casals and Cañadas, 2012). Surfactant is composed of approximately $90 \%$ lipids and $10 \%$ proteins. Phosphatidylcholine (PC) is the predominant component of surfactant lipids (Goss et al., 2013) in addition to low amounts of phosphatidylglycerol (PG), phosphatidylethanolamine (PE), phosphatidylinositol (PI), phosphatidylserine (PS), and lysophosphatidylcholine (LPC) (Goerke, 1998; Serrano and Pérez-Gil, 2006). Four known surfactant proteins (SPs) are classified into two groups: hydrophobic and hydrophilic. Hydrophobic SPs, Sftpb and Sftpc, are essential in facilitating the adsorption and stabilization of the surfactant film (Cabré et al., 2018; Curstedt et al., 1987), whereas hydrophilic SPs, Sftpa and Sftpd, are involved in the immune response to foreign entities (Crouch and Wright, 2001).

Pulmonary SP secretion is a tightly regulated process. In ATII cells, surfactant proteins are synthesized in the endoplasmic reticulum (ER) and transported to the Golgi apparatus (GA) (Brasch et al., 2004). Newly synthesized prosurfactant proteins $\mathrm{B}$ and $\mathrm{C}$ are further transported from the GA to the lamellar bodies (LBs) via multivesicular bodies (MVBs) (Matsumura et al., 2006). During the trafficking process from GA to MVBs, Sftpb and Sftpc precursors undergo glycosylation or palmitoylation and proteolytic cleavage (Guttentag, 2008; Roldan et al., 2015). Pro-Sftpb and pro-Sftpc precursors become fully mature along their trafficking route to LBs, where they then integrate into the surfactant lipid-protein complexes and, upon fusion of LBs with the plasma membrane, are secreted into the alveolar airspace (Johansson and Curstedt, 1997). Other proteins recovered in surfactant, including Sftpa and Sftpd, are secreted directly from the GA in an LB-independent manner (Froh et al., 1993; Ikegami and Jobe, 1998). Pulmonary function is dependent upon the precise regulation of alveolar surfactant. Surfactant deficiency impairs ventilation, causes lung injury, and is a major cause of mortality in premature children (Griese et al., 2015; Whitsett et al., 2015). Therefore, identification of the molecular pathways that regulate alveolar surfactant homeostasis will be a major therapeutic advance for patients with lung diseases induced by disruption of surfactant homeostasis.

GM130 was initially identified as a Golgi matrix protein localized at the $c i s$-side of the GA to maintain its structural integrity (Marra et al., 2007; Nakamura et al., 1995). Evidence collected from in vitro systems suggests that GM130 is required for ER-to-Golgi transportation, protein glycosylation, cell cycle progression, and cell migration (Baschieri et al., 2014; Wei et al., 2015; Weide et al., 2001). However, the physiological functions of GM130 in pulmonary epithelial cells have not been investigated. The results of this study demonstrated that GM130 is essential for GA structural maintenance in pulmonary ATII cells. Inactivation of GM130 resulted in inhibited SP secretion accompanied by enlarged alveolar airspace and autophagy in alveolar epithelial cells. Thus, our data demonstrated an essential role of GM130 and mammalian Golgi structure during pulmonary SP secretion and postnatal lung development.

\section{RESULTS}

\section{GM130 deficiency causes enlargement of the lung air space in mice}

Our previous work has shown that GM130 is expressed at high levels in the lung compared to other tested organs (Liu et al., 2017), which prompted us to investigate the in vivo function of GM130 during lung development. The results of histological analysis of inflated lung structures of $G M 130^{-/-}$ mice at various developmental stages indicated a considerable enlargement of the alveolar airspace in the lungs of $G M 130^{-/-}$mice at P14 and P28; however, no apparent differences between the control and $G M 130^{-1-}$ mice were observed at E18.5 (Figure 1A). The mean linear intercept (MLI), which describes the mean free distance in airspace and is a surrogate parameter for alveoli, was significantly larger in the lungs of $G M 130^{-/-}$mice than that in the control mice (Figure 1B). These findings suggest that GM130 is required for postnatal lung development in mice.

To assess whether GM130 deletion affects alveolar epithelial cell differentiation, immunofluorescence costaining was performed using antibodies against ATI and ATII cells. All analyzed alveolar epithelial markers, including Aqp5, Ager, Sftpc, and Abca3, were expressed in the lungs of $G M 130^{-/-}$mice (Figure 1C). The results of quantitative RTPCR (qRT-PCR) analysis showed comparable expression levels of ATI and ATII marker genes in $\mathrm{GM}_{3} 30^{+/+}$and $G M 130^{-/-}$mice (Figure 1D) suggesting that alveolar epithelium differentiation occurs in $G M 130^{-/-}$mice. Elastin deposition and secondary septa generation also appeared normal in the lungs of $G M 130^{-/-}$mice (Figure 1E). Because GA function is required for protein glycosylation (Puthenveedu et al., 2006), Alcian blue and periodic acid-Schiff (AB-PAS) staining was performed to detect glycoproteins in the lungs of $G M 130^{-/-}$mice. A decrease in glycoproteins was detected in alveoli of $G M 130^{-/-}$mice (Figure 1F) implying that GM130 deficiency inhibits the secretion of glycoproteins in the lungs.

\section{GM130 is required for Golgi architecture maintenance in ATII epithelial cells}

GM130 is a cis-Golgi matrix protein required for stacking of Golgi cisternae and Golgi structural maintenance (BarinagaRementeria Ramirez and Lowe, 2009); thus, we analyzed the 

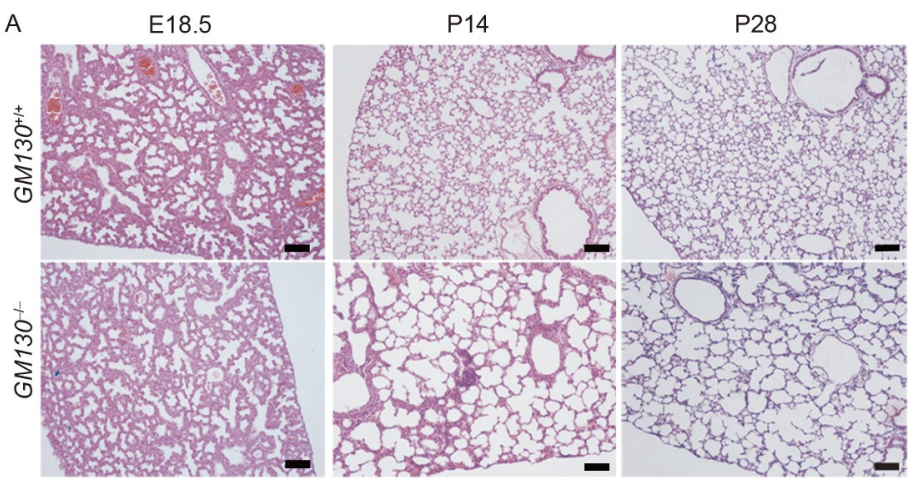

B
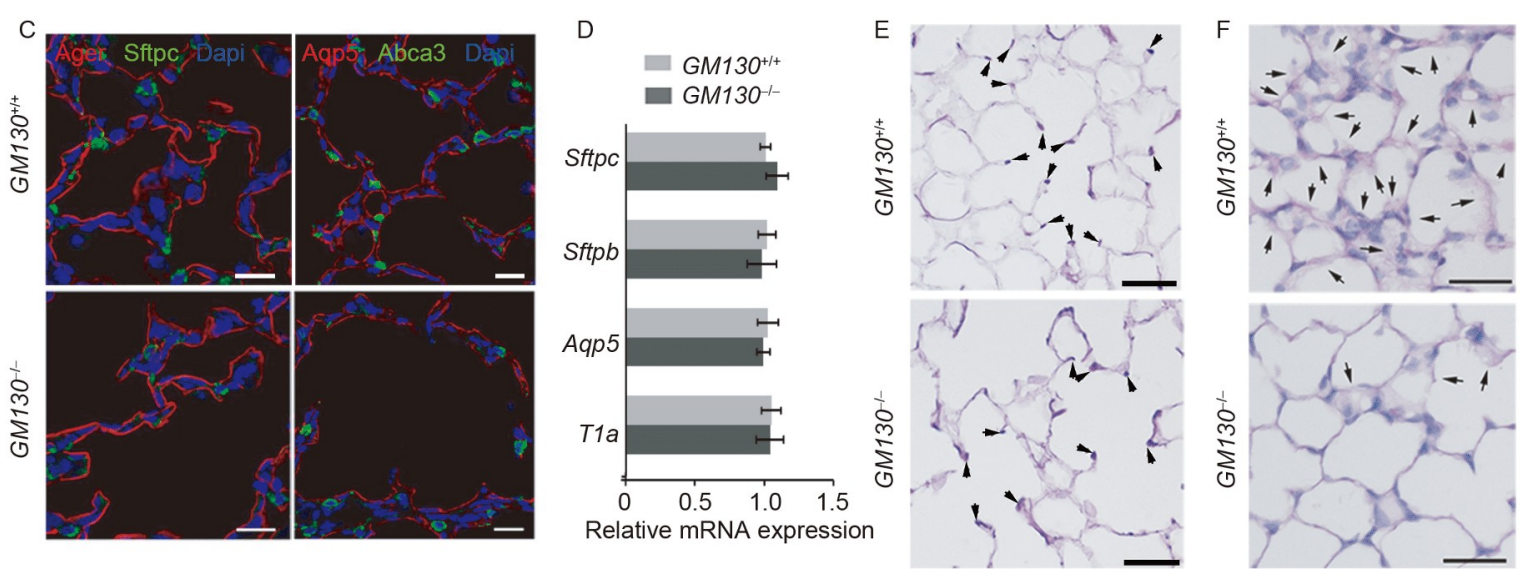

Figure 1 Loss of GM130 results in enlarged air spaces in the lungs. A, Representative images of hematoxylin and eosin (H\&E)-stained lung sections of $G M 130^{+/+}$and $G M 130^{-/-}$mice at embryonic day 18.5 (E18.5), postnatal day 14 (P14), or postnatal day 28 (P28). Scale bars: $100 \mu \mathrm{m}$. B, Multiple H\&E images of the lungs of $G M 130^{+/+}$and $G M 130^{-/-}$mice were analyzed for the MLI. $n=8$ animals per group. Data are presented as the means \pm SD. ${ }^{* *}, P<0.01$. C, Representative immunostaining images showing that both alveolar type I (Ager or Aqp5)- and type II (Abca3 or Sftpc)-specific epithelial markers were normally expressed in the lungs of $G M 130^{-/-}$mice. Scale bars: $20 \mu \mathrm{m}$. D, qRT-PCR analysis of the expression of the indicated genes in the lungs of $G M 130^{+/+}$ and $G M 130^{-/-}$mice. The data were normalized to the GAPDH expression levels. The data are represented as the means \pm SD. $n=5$ biological replicates of three independent experiments. E, Gomori aldehyde-fuchsin staining showing similar elastin deposition in the lungs of $G M 130^{+/+}$and $G M 130^{-/-}$mice. Black arrows indicate elastin. Scale bars: $20 \mu \mathrm{m}$. F, Representative Alcian blue and periodic acid-Schiff (AB-PAS) staining of lung sections showing a decrease in glycoproteins in the alveoli of $G M 130^{-/}$mice. Black arrows indicate positive signals. Scale bars: $20 \mu \mathrm{m}$.

morphology of GA in the control and $G M 130^{--}$ATII cells. In $G M 130^{+/+}$ATII cells, the data of transmission electron microscopy (TEM) revealed a well-organized ribbon-like structure of the Golgi complex with cisternae appearing in a typical arrangement of flattened saccules (Figure 2A). However, in $\mathrm{GM}_{130^{-/-}}$ATII cells, the ribbon-like structure of the Golgi complex was destroyed, and the residual cisternae consisted of disorganized vesicles and dilated tubules (Figure 2A). On the other hand, the MVB and LB, which are two other specific membranous organelles required for SP secretion in ATII cells, remained normal in $\mathrm{GM}_{130^{-/-}}$mice (Figure 2B). Thus, these observations demonstrate that GM130 is essential for GA structural maintenance in pulmonary ATII epithelial cells.

\section{GM130 absence in the pulmonary epithelium inhibits lipid secretion in alveoli}

To further investigate the role of GM130 during lung development, we conditionally inactivated GM130 in the pul- monary epithelium by generating triple-transgenic mice $G M 130^{f l / f l} ; S P C$-rtTA;TetO-Cre (referred to as $G M 130^{\text {epko }}$ ). Knockdown of GM130 in the lung tissues was detected by Western blot (Figure S1A in Supporting Information). Histological analysis showed that the alveolar airspaces were significantly increased in GM130 $0^{\text {epko }}$ mice (Figure S1B and C in Supporting Information), and the secretion of glycoproteins in $G M 130^{e p k o}$ alveoli was also decreased (Figure S1D in Supporting Information), thereby phenocopying the defects found in global GM130 knockout mice.

To investigate whether the biosynthesis and secretion of lipids are affected in GM130 knockout mice, lipidomic analyses were performed using bronchoalveolar lavage (BAL) and the remaining lung tissue. We found that most surfactant lipid components, including phosphatidylcholine (PC) and dipalmitoyl phosphatidylcholine (DPPC), which is the predominant component of $\mathrm{PC}$ in lung surfactant, were present at higher levels in the lung tissues of $G M 130^{\text {epko }}$ mice compared with those in the control mice (Figure $2 \mathrm{C}$ and $\mathrm{D}$ ). Ganglioside GM3 is a ganglioside that plays a role in the 
A
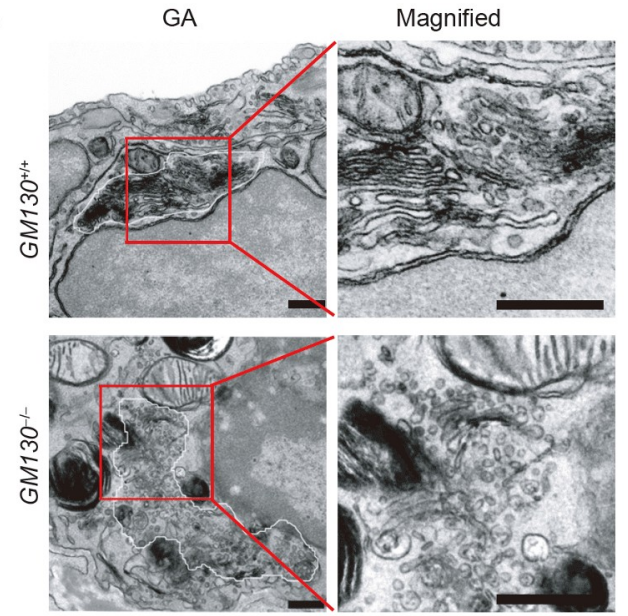

C

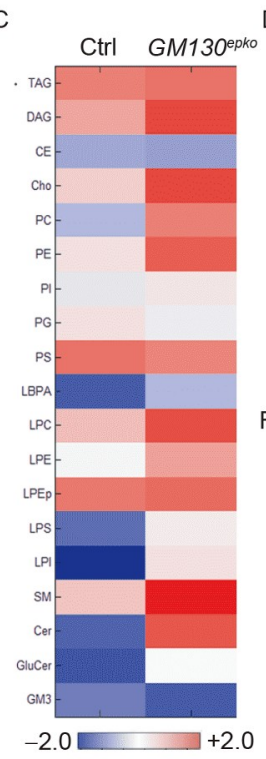

$\mathrm{D}=$

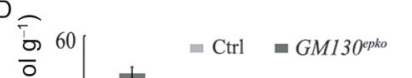

B
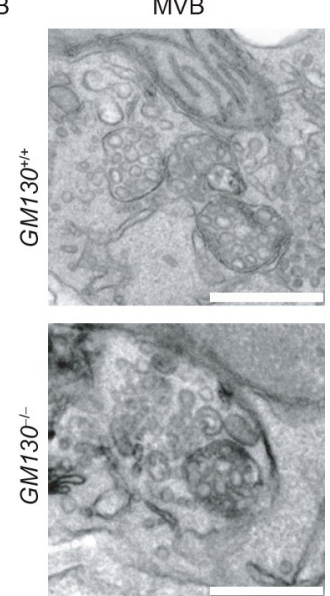

$\mathrm{E}$
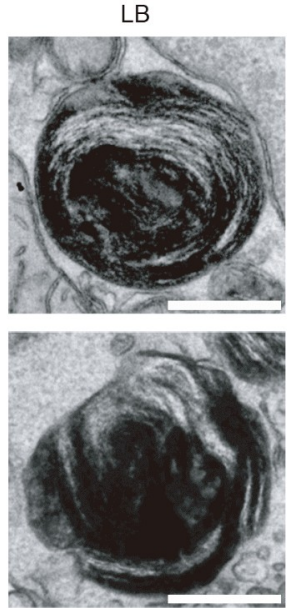

$=$

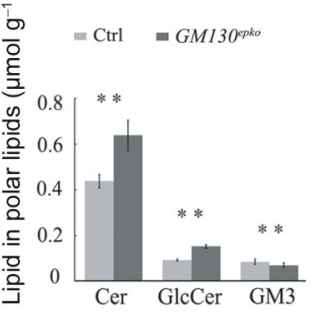

G

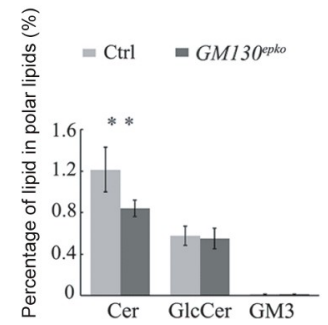

Figure 2 (Color online) Disrupted Golgi structure and impaired lipid biosynthesis and secretion in GM130 knockout mice. A and B, Representative TEM images of lung sections of $G M 130^{+/+}$and $G M 130^{-/-}$mice. White curve indicates a fragmented Golgi region in the lungs of $G M 130^{-/-}$mice. Boxed areas in low magnification images (left) are displayed at higher magnification (right). Scale bars: $0.5 \mu \mathrm{m}$. C, Heatmap generated based on the lipidomics data shows that the GM130 mutant has higher levels of most lipids in the lungs compared with those in the control. D-G, Quantification of lipid levels in lung tissue (D and $\mathrm{E}$ ) and BAL (F and $\mathrm{G}$ ) of control and $G M 130^{\text {epko }}$ mice. Data are presented as the mean $\pm \mathrm{SD} . n=4$ biological replicates per group. ${ }^{*}, P<0.05 ;{ }^{* *}, P<0.01$ (Kruskal-Wallis test). TAG, triacylglycerol; SM, sphingomyelin.

suppression of cancer development and progression; the level of GM3 was significantly decreased in the lung tissues of $G M 130^{\text {epko }}$ mice, while the levels of ceramide (Cer) and glucosylceramide (GlcCer) (Hakomori and Handa, 2015), which are the common precursors of various glycosphingolipids (van Meer and Hoetzl, 2010), were dramatically increased (Figure 2E) implying attenuated glycosphingolipid biosynthesis in the lungs of $G M 130^{\text {epko }}$ mice. In the BAL fractions, the levels of most of the lipids were similar in $G M 130^{\text {epko }}$ and control mice. The levels of a few lipid class components, including PE, LPC, SM, and Cer, were significantly decreased (Figure $2 \mathrm{~F}$ and $\mathrm{G}$ ), implying compromised secretion of these lipid components in the absence of GM130 in the pulmonary epithelium. Overall, these data imply that GM130 loss selectively inhibits lipid biosynthesis and secretion in pulmonary epithelial cells.

\section{GM130 regulates the secretion of surfactant proteins}

Because of the differences in the morphology of the Golgi complex in $\mathrm{GM}_{130^{-/-}}$cells, it was of interest to investigate whether SP secretion is affected in the lungs of GM130 knockout mice. The BAL fractions from the control and $G M 130^{e p k o}$ mice were analyzed to detect the secreted protein levels. Coomassie blue staining of BAL proteins identified a specific band that was abundant in control mice but was barely detectable in the $G M 130^{\text {epko }}$ BAL samples (Figure 3A, left). Mass spectrometry analysis showed that this band contains unique mature $\mathrm{Sftpb}$ and mature $\mathrm{Sftpc}$ peptides (Figure 3A, right), implying that $\mathrm{Sftpb}$ and Sftpc secretion is 

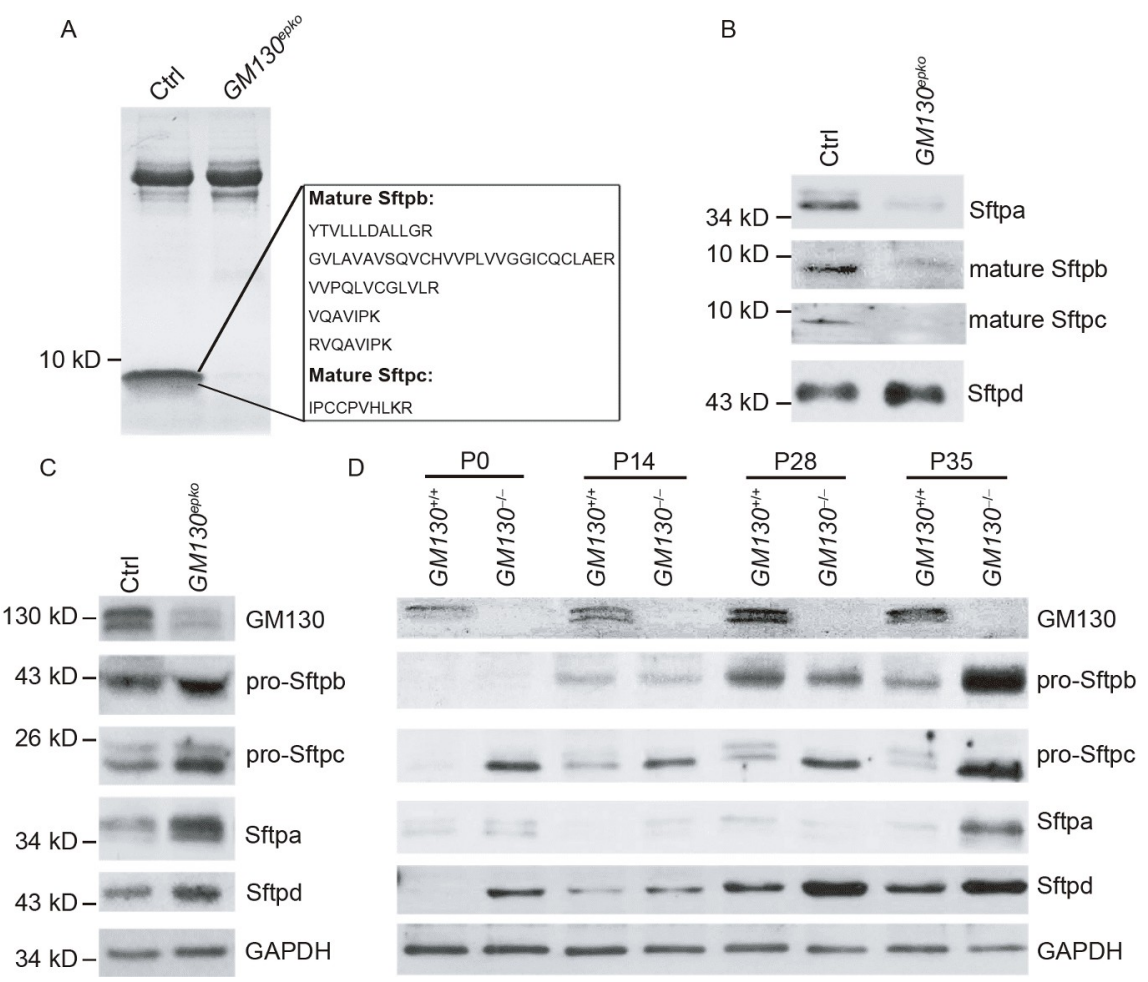

Figure 3 Loss of GM130 inhibits the secretion of SPs in pulmonary epithelial cells. A, Inhibition of secretion of mature Sftpb and mature Sftpc in $G M 130^{e p k o}$ mice. Left panel, Coomassie blue staining of BAL proteins of the control and $G M 130^{e p k o}$ mice. Right panel, unique peptides of mature Sftpb and mature Sftpc identified by mass spectrometry analysis. B and C, Western blot analysis of SP expression levels in BAL (B) and lung tissues (C) of the control and $G M 130^{\text {epko }}$ mice. D, Western blot analysis of SP expression levels in the lung tissues of $G M 130^{+/+}$and $G M 130^{-/-}$mice at the indicated developmental stages. GAPDH was used as an internal control.

impaired in $G M 130^{\text {epko }}$ mice. A decrease in the expression of mature Sftpb and Sftpc in BAL was confirmed by Western blot (Figure 3B). Moreover, an increase in Sftpd expression and a decrease in Sftpa expression were detected in $G M 130^{e p k o}$ mice (Figure 3B). On the other hand, the levels of all tested SPs, including pro-Sftpb, pro-Sftpc, Sftpa, and Sftpd, were dramatically increased in the lung tissues of $G M 130^{\text {epko }}$ mice (Figure 3C). Interestingly, Sftpa or pro$\mathrm{Sftpb}$ accumulation was not detected in the lungs of $\mathrm{GM} 130^{-/-}$ mice until P35, whereas the levels of pro-Sftpc and Sftpd were considerably increased in $G M 130^{-/-}$mice starting at P0 (Figure 3D). Overall, these experiments demonstrate that GM130 deficiency selectively inhibits SP secretion in the pulmonary epithelium.

\section{Deletion of GM130 selectively inhibits SP trafficking in MEFs}

To confirm impaired SP secretion, we constructed green fluorescent protein (GFP)-tagged SP vectors and examined the localization and transport of these GFP-SP fusion proteins in mouse embryonic fibroblasts (MEFs). Immunofluorescence staining showed that $\mathrm{Sftpc}$ protein colocalized with the GFP signal (Figure S2A in Supporting Information) suggesting that GFP fluorescence represents SP localization in transfected MEFs. We then assessed SP location in MEFs $24 \mathrm{~h}$ after transfection. Scattered punctate distribution of GFP-SP was detected in the cytoplasm of $\mathrm{GM} 30^{+/+} \mathrm{MEFs}$; however, the GFP signals of Sftpb and Sftpc showed very prominent cluster-like aggregates in $G M 130^{-/-}$MEFs (Figure S2A and B in Supporting Information). Similar structures and diffuse cytoplasmic signals were observed in GFP-Sftpatransfected $\mathrm{GM}_{130^{-/}}$cells (Figure S2B in Supporting Information), whereas GFP-Sftpd-transfected cells showed punctate cytoplasmic localization similar to that in the control cells (Figure S2B in Supporting Information). Since SPs are transported from the ER to GA, we determine whether these aggregated SPs (Sftpa, Sftpb, and Sftpc) are trapped in the ER or in the GA. Immunostaining of Sec61b, Golgin-84, or TGN38 showed that the diffuse cytoplasmic and aggregated cluster-like GFP-Sftpa signals were colocalized with the ER marker Sec61b in GM130 $0^{-/-}$MEFs (Figure S3A in Supporting Information), whereas aggregated GFP-Sftpb and GFP-Sftpc were predominantly localized in the GA (Figure 4A and B). Finally, we analyzed the transport by assaying GFP-SP localization at various time points after the transfection. In the control cells, cytoplasmic GFP-SP signals became scattered, had a punctate distribution, started to fade $24 \mathrm{~h}$ after the transfection, and almost disappeared at $48 \mathrm{~h}$ suggesting GFP-SP secretion within the cells (Figure 

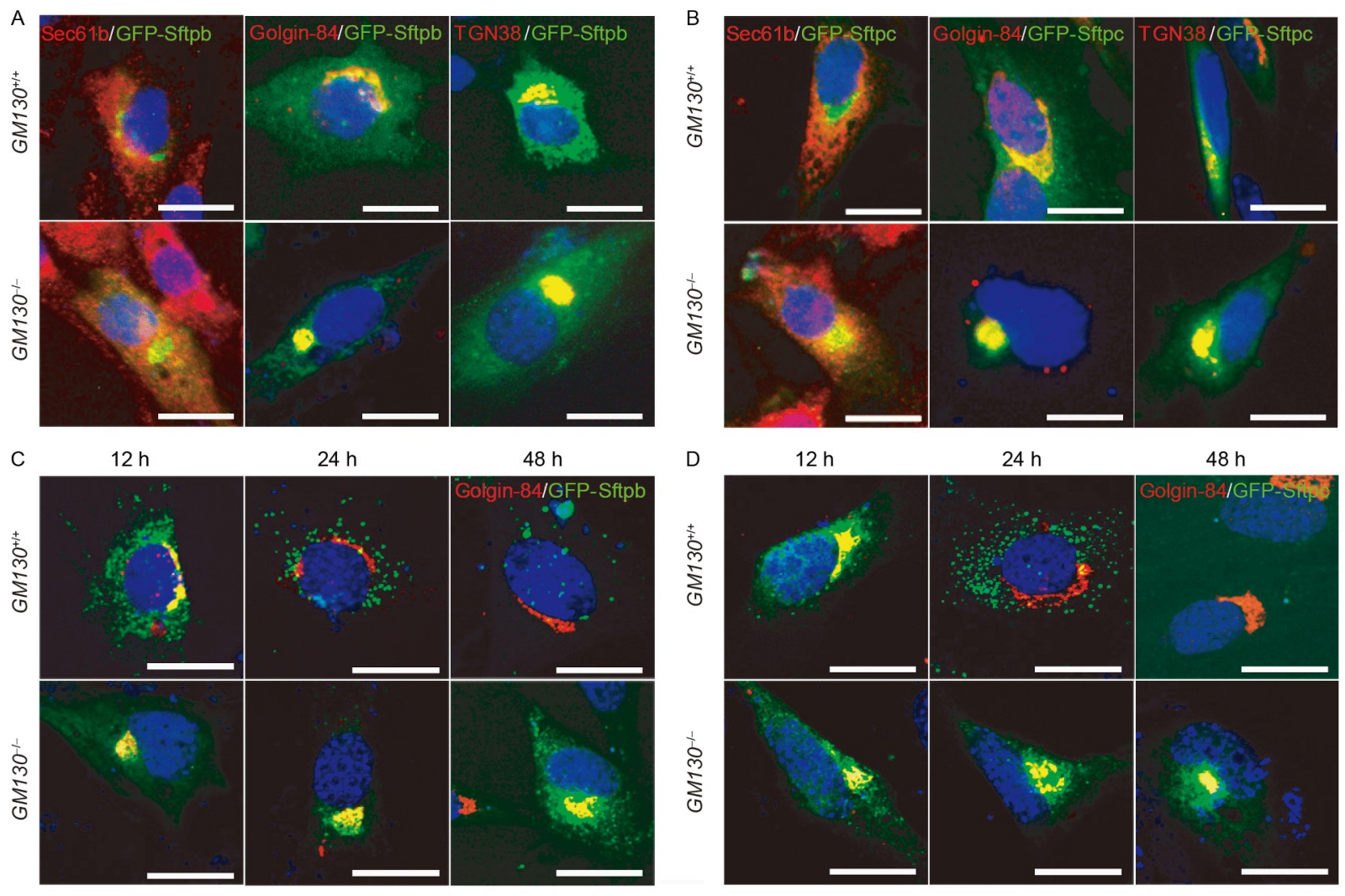

$24 \mathrm{~h}$

$48 \mathrm{~h}$

E

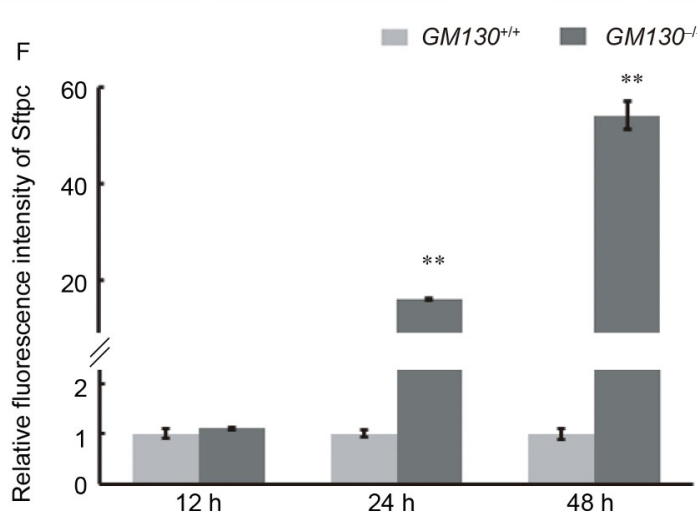

Figure 4 Trapping of GFP-Sftpb and GFP-Sftpc in GA in GM130 ${ }^{-/}$MEFs. A and B, Representative immunofluorescence images showing the subcellular localization of GFP-Sftpb (A) and GFP-Sftpc (B) in MEFs generated from $G M 130^{+/+}$and $G M 130^{-/-}$mice. MEFs were transfected with GFP-Sftpb (A) or GFP-Sftpc (B) constructs, and the staining was performed $12 \mathrm{~h}$ later using anti-Sec61b (ER marker), anti-Golgin-84 (cis-Golgi marker), or anti-TGN38 (trans-Golgi marker) antibodies. Scale bars: $25 \mu \mathrm{m}$. C and D, Expression patterns of GFP-Sftpb (C) and GFP-Sftpc (D) in MEFs 12, 24 , and $48 \mathrm{~h}$ after the transfection. Cells were immunostained with anti-Golgin-84 antibody to label GA. Scale bars: $25 \mu \mathrm{m}$. E and F, of Sftpb (E) and Sftpc (F) fluorescence intensity in MEFs 12,24 , and $48 \mathrm{~h}$ after the transfection. Values are the mean $\pm \mathrm{SD} ; n>20$ cells from 3 independent experiments; $* *, P<0.01$ (Student's $t$-test).

4C and D; Figure S3B and C in Supporting Information). In contrast, GFP-SPs were trapped and accumulated either in the ER (GFP-Sftpa) or GA (GFP-Sftpb and GFP-Sftpc) in GM130 $0^{-1-}$ MEFs in a time-dependent manner (Figure 4C-F; Figure S3B in Supporting Information). Moreover, Western blot analysis demonstrated a significant increase in fulllength GFP-pro-Sftpb and GFP-pro-Sftpc expression in GM130 $0^{-1-}$ MEFs (Figure S3D in Supporting Information) consistent with the results obtained in the lung tissues of $G M 130^{-/-}$mice, which showed the accumulation of pro$\mathrm{Sftpb}$ and pro-Sftpc proteins (Figure 3C and D). Overall, these results imply that GM130 is essential for Sftpa, Sftpb, and Sftpc transport within MEFs.

\section{GM130 is required for SP trafficking in lung ATII cells}

To determine whether GM130 is required for SP secretion in vivo, we analyzed the subcellular localization and expression of SPs in lung ATII cells by coimmunofluorescence staining. Consistent with the observations in $G M 130^{-/-} \mathrm{MEFs}$, significantly increased expression of all SP members was observed in ATII cells in the absence of GM130 (Figure 5A-C; 

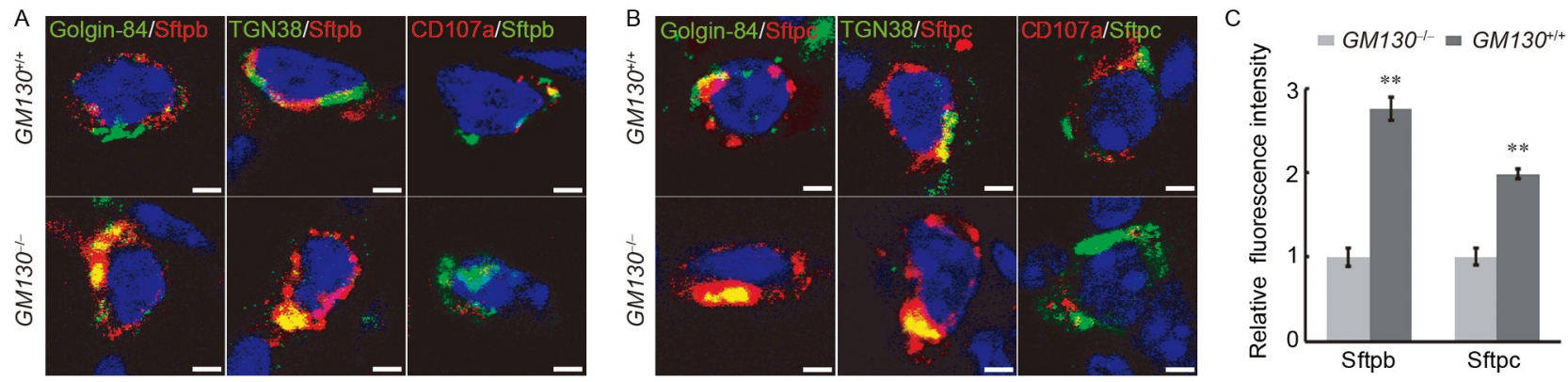

Figure 5 GM130 knockout induces Sftpb and Sftpc accumulation in GA in ATII epithelial cells. A and B, Representative double-immunostaining images of P28 lung sections generated using indicated antibodies. Note that Sftpb (A) and Sftpc (B) are colocalized with the cis-Golgi marker Golgin-84 and transGolgi marker TGN38 in ATII epithelial cells and are not colocalized with the lamellar body marker CD107a. Scale bars: $2 \mu \mathrm{m}$. C, Quantification of endogenous Sftpb and Sftpc fluorescence intensity in $G M 130^{+/+}$and $G M 130^{-/-}$ATII cells. Values are the mean \pm SD. $n>20$ cells from 3 independent experiments; **, $P<0.01$ (Student's $t$-test).

Figure S4A-C in Supporting Information). Except for Sftpd, which displayed a scattered cytoplasmic distribution similar to that in the control cells, other SPs, including Sftpa, Sftpb, and Sftpc, were aggregated on a large-scale with concentrated fluorescence signals in $G M 130^{-/}$ATII cells (Figure 5A and B; Figure S4A in Supporting Information). Subsequent analysis showed that these aggregates are colocalized with ER markers (for Sftpa) (Figure S4A in Supporting Information) or GA markers (for Sftpb and Sftpc) (Figure 5A and B). Notably, a small fraction of Sftpb or Sftpc was detected in the fragments of GA and LBs (Figure 5A and B), implying a normal GA-to-LB transport. Overall, these data suggest that GM130 inactivation impairs pulmonary surfactant secretion in vivo.

\section{Deletion of GM130 triggers autophagy in pulmonary epithelial cells}

The data derived from various sources suggested that Golgi fragmentation causes the accumulation of misfolded proteins and induces ER stress, and prolonged ER stress may trigger autophagy and apoptosis (Joachim et al., 2015; Lawson et al., 2011). To assess whether ER stress is induced in the lungs of GM130 $0^{-1-}$ mice, we assayed the expression of ER stress proteins by Western blot. As shown in Figure 6A, the protein levels of inositol-requiring enzyme 1 (IRE-1), binding immunoglobulin protein (Bip), and the activated form of X-box binding protein (XBP1) were not increases in the lungs of $G M 130^{-/-}$mice. Moreover, no significant increase in the percentage of apoptotic ATII cells was observed in the lungs of $\mathrm{GM}_{130^{-/-}}$mice (Figure 6B and C) implying that GM130 deletion did not induce ER stress or ATII apoptosis.

To determine whether GM130 loss is associated with autophagy, Western blot was used to detect the conversion of microtubule-associated proteins 1 light chain 3-I (LC3-I) to LC3-II in GM130 $0^{+/+}$and GM130 $0^{-/}$mice. LC3-I and LC3-II are the cellular forms of LC3 protein; LC3-I is the cytoplasmic form processed by enzymatic cleavage into LC3-II, which is the autophagosome membrane-bound form
(Klionsky et al., 2016). Therefore, the level of LC3-II protein is commonly used to monitor the formation of autophagosomes. GM130 deletion caused a significant increase in the levels of the LC3-II protein in a time-dependent manner (Figure 6D and E), and an increase in the ratio of $\mathrm{LC} 3-\mathrm{II}^{+}$ cells was observed in the lungs of $G M 130^{-/-}$mice by immunofluorescence assay (Figure $6 \mathrm{~F}-\mathrm{H}$ ). In addition, autophagosome formation was detected in $G M 130^{-/-}$lung ATII cells (Figure 6I). Overall, these results suggest that loss of GM130 triggers autophagy in pulmonary epithelial cells.

We then examined whether autophagy contributes to the impaired SPs secretion in GM130-deficient mice. MEFs were treated with rapamycin (RAPA, an immunosuppressant that induces autophagy by inhibiting mTOR protein kinase), chloroquine (CQ, a lysosomal inhibitor that blocks autophagy by inhibiting lysosomal degradation) or a typical PI3K/ Akt pathway inhibitor wortmanine (Wort, inhibition of autophagosomes formation). As previously reported (Klionsky et al., 2016), an increased expression of LC3 in RAPA- and CQ-treated cells, while a decreased LC3 expression, were detected in Wort- treated $G M 130^{+/+}$and $G M 130^{-/-}$MEFs (Figure S5A in Supporting Information). However, after $48 \mathrm{~h}$ of GFP-SPs transfection and chemical treatment, GFP-SPs signals were barely detected within $G M 130^{+/+}$cells, we still observed aggregated cluster-like signals (GFP-Sftpb and GFP-Sftpc) and scattered punctate signals (GFP-Sftpd) in GM130 $0^{-/-}$MEFs (Figure S5B-D in Supporting Information), the same GFP-SPs expression pattern as in DMSOtreated $G M 130^{-/-}$cells. Moreover, knock out of autophagyrelated gene 5 (Atg5) by siRNA also had very little effect on GFP-SPs secretion in MEFs (Figure S6A-D in Supporting Information). These observations indicate that the effects of GM130 on autophagy are independent of its effects on SPs secretion.

\section{Surfactant repletion partially rescues the enlarged air- space defects in GM130-deficient mice}

To determine whether a decrease in SP secretion contributes to 

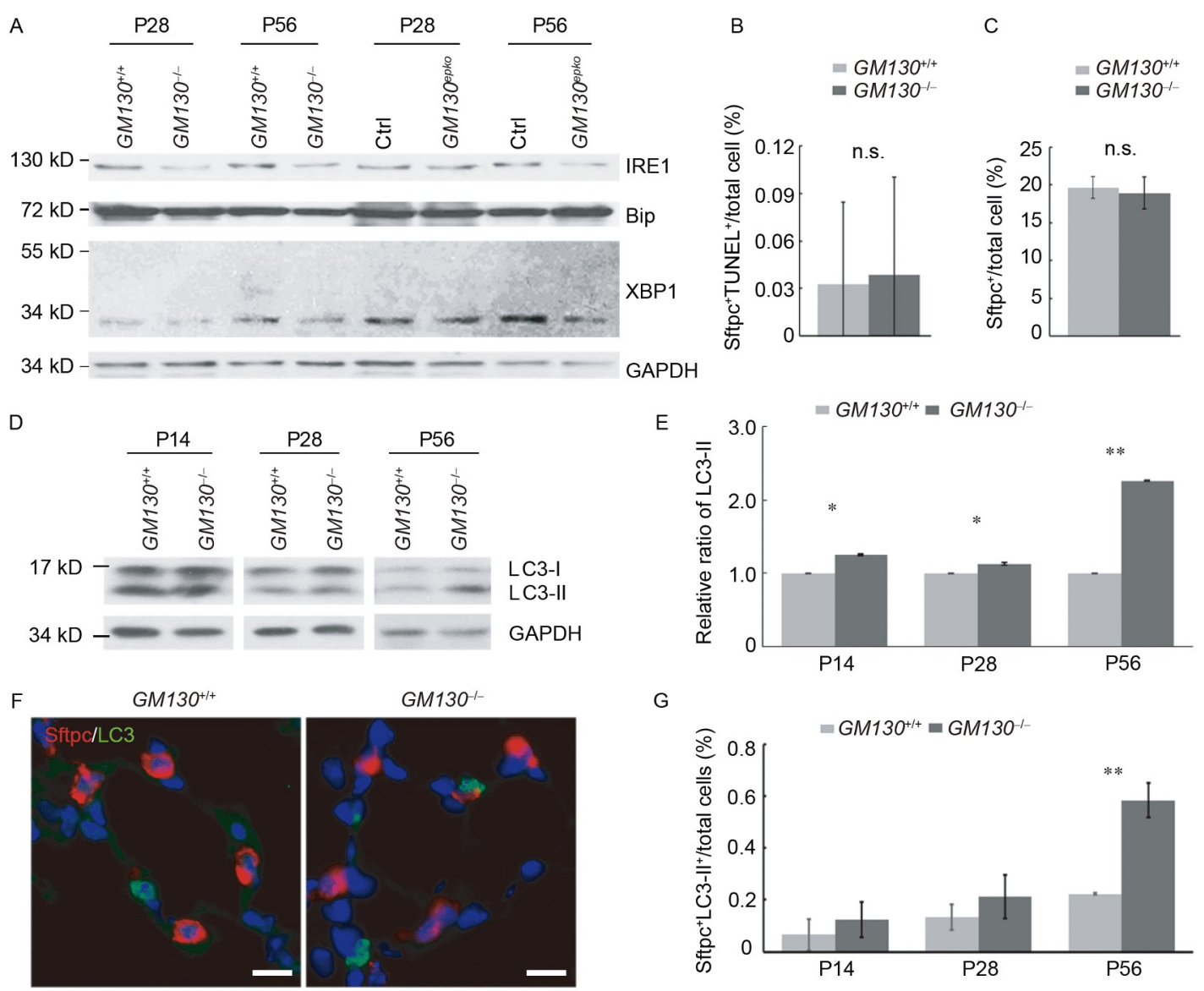

G
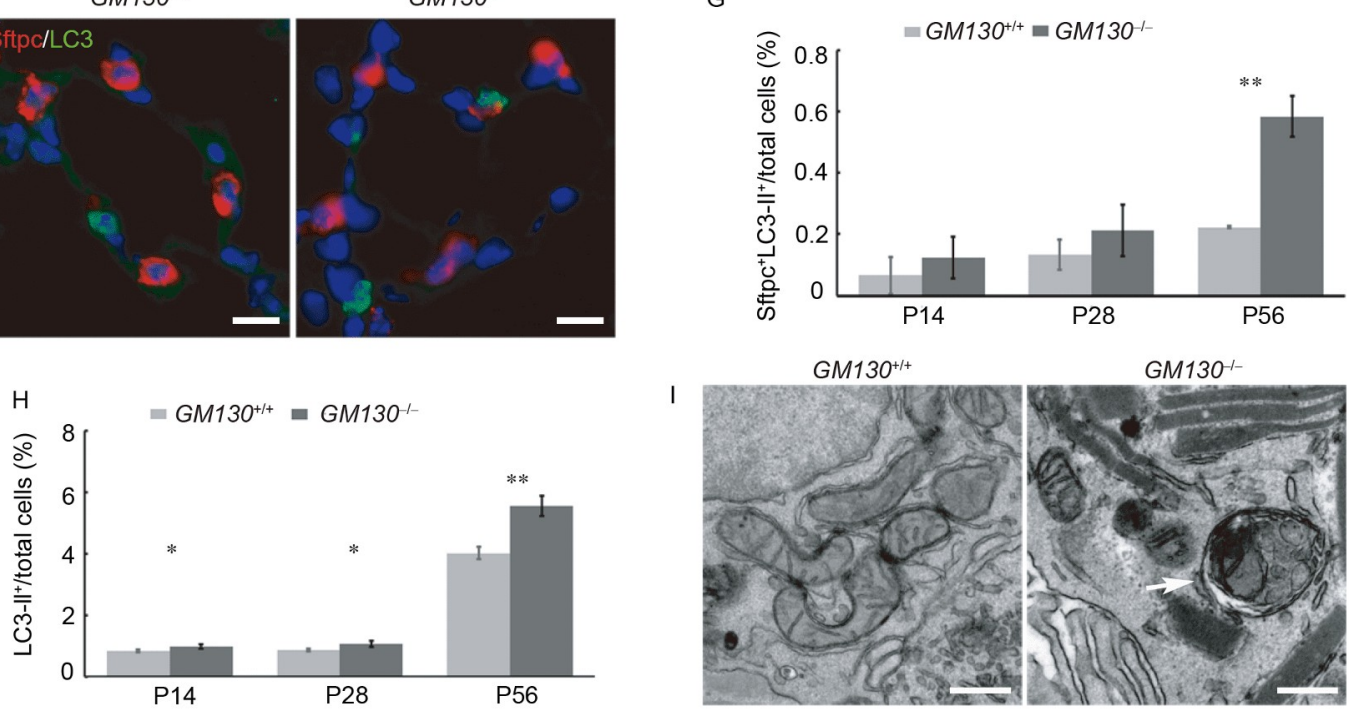

Figure 6 Loss of GM130 triggers autophagy in lung ATII cells. A, Western blot analysis of ER stress-associated proteins. XBP1 (inactive XBP1, 33 kD; active XBP1, $54 \mathrm{kD})$, Bip, and IRE1. B and C, Quantification of Sftpc $\mathrm{TUNEL}^{+}$cells (B) and Sftpc cells $(\mathrm{C})$ in the lung sections of $\mathrm{TM}^{+} 130^{+/+}$and $\mathrm{GM}^{+} 30^{-/-}$ mice. $n=4$ animals per group. Data are presented as the mean \pm SD. n.s., not significant. D, Representative Western blot images showing the expression levels of LC3-I and LC3-II in the lung tissues of $\mathrm{GM}_{130^{+/+}}$and $G M 130^{-/-}$mice at the indicated developmental stages. E, Quantification of LC3-II protein levels. Data was normalized to GAPDH expression levels. Values are the mean \pm SD. $n=5$ biological replicates; $*, P<0.05$; $* *, P<0.01$ (Student's $t$-test). F, Representative immunostaining images showing an increase in $\mathrm{LC}^{+}$cells in the lungs of GM130 ${ }^{-/-}$mice. Scale bars: $10 \mu \mathrm{m}$. $\mathrm{G}$ and $\mathrm{H}$, Quantification of $\mathrm{Sftpc}^{+} \mathrm{LC} 3-\mathrm{II}^{+}$cells $(\mathrm{G})$ and LC3-II ${ }^{+}$cells $(\mathrm{H})$. Data are presented as the mean $\pm \mathrm{SD} . n=5$ animals per group. *, $P<0.05$; **, $P<0.01$ (Student's $t$-test). I, Representative TEM images of the lungs of $G M 130^{+/+}$and $G M 130^{-/-}$mice. White arrowhead indicates the autophagosome. Scale bars: $0.5 \mu \mathrm{m}$.

the developmental airspace defects in GM130-deficient mice, we administered CALSURF (calf pulmonary surfactant for injection) to neonatal $G M 130^{+/+}$and $G M 130^{-/-}$mice. CALSURF is a natural bovine lung extract containing phospholipids, cholesterol, triglycerides, free fatty acids, and surfactantassociated proteins (Sftpb and Sftpc). CALSURF is commonly used in clinical practice for the treatment and prevention of neonatal respiratory distress syndrome (RDS). In our experiment, newborn mice were treated intratracheally with CALSURF for 3 days, and the control groups were treated with an equivalent volume of PBS. Administration of CAL-
SURF to $G M 130^{+/+}$mice had no effect on alveolar airspaces (Figure 7A and B). However, treatment of $\mathrm{GM} 30^{-/-}$mice with CALSURF significantly decreased airspaces compared to that in the PBS-treated control groups (Figure 7A and B) suggesting that a decrease in SP secretion contributes to enlarged lung airspace in GM130-deficient mice.

\section{DISCUSSION}

The results of this study demonstrate that cis-Golgi matrix 


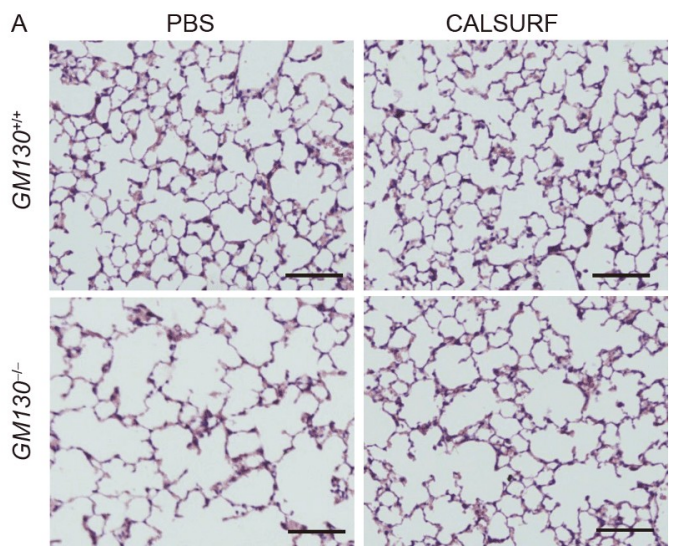

B

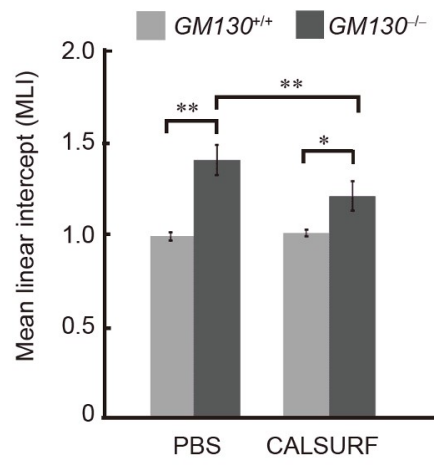

Figure 7 (Color online) PS administration partially rescues the enlarged alveolar airspace developmental defects in $G M 130^{-/-}$mice. A, Representative images of H\&E-stained lung sections showing that an increase in the alveolar spaces in $G M 130^{-/}$mice is partly rescued after PS treatment. Scale bars: $100 \mu \mathrm{m}$. B, Quantification analysis of MLI in $G M 130^{+/+}$and $G M 130^{-/-}$mice. Data are presented as the mean \pm SD. *, $P<0.05$; **, $P<0.01$ (one-way ANOVA with Tukey's test).

protein GM130 is required for maintenance of the Golgi ribbon-like structure and SP secretion in pulmonary epithelial cells. GM130 deletion resulted in the fragmentation of the GA structure and accumulation of three SPs within lung ATII cells, whereas Sftpd secretion was not impaired (Figure 8). As a consequence of disrupted SP secretion, GM130 knockout mice manifest the enlarged pulmonary airspace phenotype. Thus, our findings demonstrate that mammalian GA is an indispensable organelle that selectively influences SP secretion.

GA is a highly organized dynamic organelle at the center of the intracellular secretory pathway. Posttranslational modification and trafficking of secretory cargo proteins and lipids are the most important roles of GA (Wong and Munro, 2014). Basic structure of GA is represented by a stack of closely aligned flattened cisternae (Ladinsky et al., 1999). Structural integrity of GA is maintained by a family of Golgi matrix proteins (Munro, 2011), microtubule-based proteins (Allan et al., 2002; Marra et al., 2007), and vesicle-trafficking proteins (Martínez-Alonso et al., 2013; Yeong, 2013). Golgi dynamics and function have been extensively studied using in vitro models (Helenius and Aebi, 2001; van Meer, 1998; Paladino et al., 2014); however, the mechanisms of Golgi-dependent secretion and regulation of the stacked Golgi structure and function in mammals are poorly understood. In the present study, we provide evidence that GM130 deficiency leads to impaired SP secretion as a consequence of the disruption of the GA ribbon-like structure. GM130 knockout mice manifest epithelial cell autophagy and enlarged airspace phenotypes. To the best of our knowledge, our study is the first to identify an intriguing molecular mechanism of the effect of GM130 on ATII cell function mediated by the control of the surfactant protein concentration during postnatal lung development.

Our finding that GA fragmentation induced by GM130 deficiency selectively blocks SP secretion is unexpected.
Sftpa was the only SP trapped in the ER suggesting that its transport from the ER to GA requires GM130. D'Angelo et al. (2009) reported that the GM130-GRASP65 complexes regulate ER-to-GA transport through certain C-terminal valine motifs (C-TVM)-containing cargo molecules. However, Sftpa does not contain C-TVM suggesting that an alternative mechanism is involved in the ER-to-GA transport of Sftpa in ATII cells. It is unclear why Sftpa is trapped in the ER, while Sftpd is transported normally. This phenomenon may be explained by synergistic transport of Sftpa together with lipids (DPPC, phosphatidylcholine, etc.) (Froh et al., 1993; Qua Hiansen et al., 2015), and Sftpd does not require surfactant lipids for intracellular processing and transport. Both Sftpb and Sftpc proteins are posttranslationally modified in the GA. Pro-Sftpb is glycosylated at $\mathrm{Asn}^{129}$ and $\mathrm{Asn}^{311}$ (Guttentag, 2008), and pro-Sftpc is dipalmitoylated at $\mathrm{Cys}^{28}$ and $\mathrm{Cys}^{29}$ (D'Angelo et al., 2009). Notably, previous studies have demonstrated that these modifications are necessary for intracellular protein transport because their absence inhibits the enzymatic cleavage of pro-SPs into mature Sftpb and Sftpc (Baumgart et al., 2010; ten Brinke et al., 2001). In GM130-deficient MEFs and ATII cells, full-length pro-proteins accumulated in the GA implying that Sftpb and Sftpc are trapped in the GA before cleavage. The accumulation of Sftpb and Sftpc can be explained by failed protein modifications due to structural disruption of GA; thus, unmodified pro-proteins cannot be transported from the GA to MVBs and cannot be enzymatically cleaved to produce mature proteins. This hypothesis is supported by the fact that knockdown of GM130 in HeLa cells disturbs the uniform distribution of Golgi enzymes and inhibits the glycosylation of secretory proteins (ten Brinke et al., 2002; Puthenveedu et al., 2006). Alternatively, it is possible that pro-proteins were properly modified, but the GA-to-MVB transport is impaired. Additional studies are needed to investigate these possibilities. 


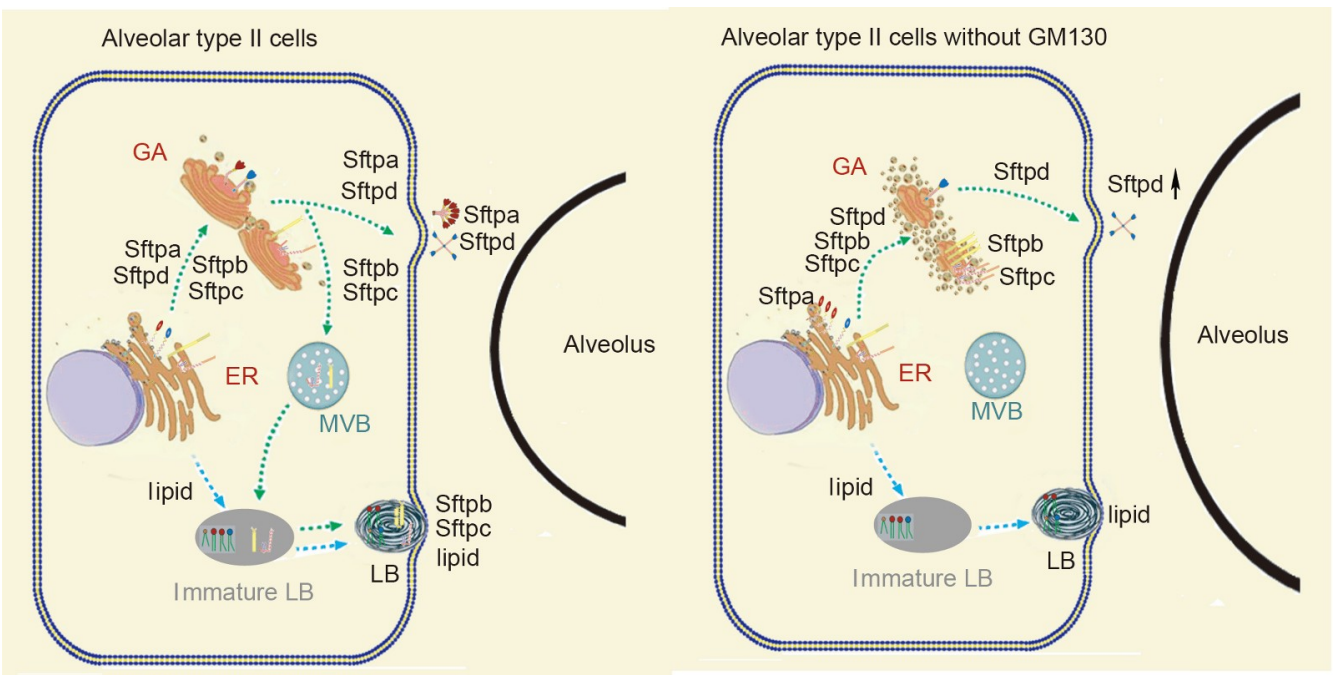

Figure 8 (Color online) Scheme of the effects of GM130 on the morphology of Golgi apparatus and SP secretion. In mature ATII cells (left), SPs are transported from the ER to GA. Sftpb and Sftpc are further transported from the GA to LB via MVBs and secreted by alveoli with lipid components. Sftpa and Sftpd are secreted directly through the GA in an LB-independent manner. In GM130-deficient ATII cells (right), the GA structure is fragmented, and SP secretion is compromised. Specifically, Sftpa accumulates in the ER, and Sftpb and Sftpc are trapped in the GA, whereas Sftpd secretion is increased. Disrupted SPs and lipid secretion are likely to contribute to the enlarged airspace phenotype in GM130 knockout mice.

In the adult lung, alveolar surfactants are tightly regulated and maintained through the balance of synthesis, secretion, and recycling by ATII cells (Wang et al., 2019). Alterations in SP concentration or function are often associated with severe respiratory pathologies, such as RDS (Orgeig et al., 2010; Whitsett et al., 2015). Previous studies suggested that GA is involved in SP secretion because disruption of the GA structure by brefeldin A completely blocks SP transport in cultured ATII cells (Wang et al., 2007). However, due to the lack of corresponding animal models, the physiological effect of GA structure on SP secretion, ATII cell function, and pulmonary development remains unknown. The results of this study demonstrate that disruption of the mammalian GA structure induced by GM130 deficiency selectively inhibits SP secretion in vivo. Moreover, GM130 knockout mice show RDS-like phenotypes, such as reduced SP secretion and enlarged airspaces. Treatment of $G M 130^{-/-}$mice a drug used in clinical practice for RDS therapy significantly rescues the enlargement of airspaces. These discoveries provide new insight into the significance of GA in mammals. Therefore, GM130 knockout mice may be a potentially valuable animal model for investigation of RDS.

\section{MATERIALS AND METHODS}

\section{Animals}

$G M 130^{f l / f l}$ and $G M 130^{-/-}$mice were generated as described previously (Liu et al., 2017). Lung epithelial-specific GM130 knockout mice $\left(G M 130^{\text {epko }}\right)$ were generated by crossing $G M 130^{f l f l}$ mice with SPC-rtTA (Tichelaar et al., 2000) and
TetO-Cre mice (Sauer, 1998). Administration of doxycycline started from E6.5 and ended by feeding pregnant mice $625 \mathrm{mg} \mathrm{kg}^{-1}$ doxycycline-containing food. A total of 3 doses of CALSURF (NMPN H20052128; $100 \mu \mathrm{g} \mathrm{kg}^{-1}$ body weight) were used for the treatment of $\mathrm{GM} 3 \mathrm{O}^{+/+}$and $G M 130^{-/-}$mice. The first dose was given at P0, and the following 2 CALSURF doses were given at $24 \mathrm{~h}$ intervals on subsequent days. All C57BL/6 mice used in this study were genotyped by genomic DNA PCR using the primers listed in Table S1 in Supporting Information.

\section{Immunofluorescence staining, imaging, and quantifica- tion}

Immunofluorescence staining was performed as described previously (Li et al., 2018). Briefly, samples were blocked in PBS containing 5\% BSA (Amersco, USA) and 2\% normal goat serum (NGS, Cell Signaling Technology, USA) for $2 \mathrm{~h}$ at room temperature. After blocking, the samples were incubated with primary antibodies overnight at $4^{\circ} \mathrm{C}$ followed by $1 \mathrm{~h}$ incubation with appropriate secondary antibodies and counterstaining with DAPI (Beyotime Biotechnology, Shanghai, China). The antibodies used in the study are listed in Table S2 in Supporting Information. Images were acquired using a Leica TCS SP8 confocal microscope. ImageJ software was used for quantification of fluorescence intensity.

\section{LC MS/MS analysis and Western blotting}

MS/MS was performed as described previously (Li et al., 2015). Briefly, specific protein bands were cut for in-gel 
trypsin digestion, and the resulting peptides were subjected to LC MS/MS (liquid chromatography and tandem mass spectrometry) analysis. The acquired MS/MS spectra were extracted and analyzed. The identified pro-Sftpb and proSftpc peptides were further validated by manual inspection of their MS/MS spectra. Western blotting was performed as described previously (Ying et al., 2015) using antibodies listed in Table S2 in Supporting Information.

\section{Plasmid construction and MEF transfection}

To express SPs with N-terminal GFP, full-length mouse SP cDNAs were cloned into the pEGFP-N3 plasmid. The primer sequences used in the study are listed in Table S1 in Supporting Information. Transfections were performed using Lipofectamine 2000. MEFs harvested from $\mathrm{GM}_{130^{+/+}}$and GM130 ${ }^{-/-}$mice were incubated with the transfection reagent and DNA for $5 \mathrm{~h}$, washed and cultured in DMEM supplemented with $10 \%$ fetal bovine serum medium in an incubator at $37^{\circ} \mathrm{C}$ and $5 \% \mathrm{CO}_{2}$ for the indicated time periods. To induce or block autophagy, the cells transfected with GFP-SPs were cultured with medium containing $5 \mathrm{mmol} \mathrm{L}^{-1}$ rapamycin (Solarbio, Beijing, China), $50 \mathrm{nmol} \mathrm{L}^{-1}$ chloroquine (Sigma-Aldrich, USA) or $100 \mathrm{nmol} \mathrm{L}^{-1}$ wortmanine (Meilunbio, Dalian, China) for $48 \mathrm{~h}$. The mouse Atg5 siRNA and scramble siRNA were produced by GenePharma Company (Shanghai, China). The target sequence for Atg5 as follows: 5'-GACGUUGGUAACUGACAAATT-3' and 5'-UUUGUCAGUUACCAACGUCTT- ${ }^{\prime}$. Cells were fixed with $4 \%$ paraformaldehyde for $20 \mathrm{~min}$ before the immunofluorescence staining assay.

\section{Gomori aldehyde-fuchsin staining and $H \& E$ staining}

For Gomori aldehyde-fuchsin staining, paraffin lung sections ( $5 \mu \mathrm{m}$ thickness) were deparaffinized and rehydrated. The sections were oxidized for $5 \mathrm{~min}$ in 5\% potassium permanganate, incubated with $2 \%$ oxalic acid, blanched for $2 \mathrm{~min}$, and dipped in $70 \%$ alcohol for $1 \mathrm{~min}$. Finally, the samples were stained in Gomori aldehyde-fuchsin staining buffer for $15 \mathrm{~min}$. H\&E staining was performed as described previously (Ying et al., 2015).

\section{Electron microscopy analysis}

Mice were perfused with $1 \%$ paraformaldehyde and $2.5 \%$ glutaraldehyde, and the tissues were fixed in $2.5 \%$ glutaraldehyde and then in $1 \% \mathrm{OsO}_{4}$ for $2 \mathrm{~h}$ on ice; the samples were dehydrated in graded acetone solutions and embedded in Embed 812. Ultrathin sections ( $65 \mathrm{~nm})$ were stained with $2 \%$ uranyl acetate for $30 \mathrm{~min}$ and lead citrate for $10 \mathrm{~min}$. Samples were imaged using a $120 \mathrm{kV}$ electron microscope (JEM America Corp., USA).

\section{Collection of BAL fluid and lipidomic analyses}

To retrieve BAL fluid, eight-week-old mice were anesthetized and perfused with PBS; tracheas were exposed and cannulated with a blunt needle. BAL was performed by injecting $0.75 \mathrm{~mL}$ of PBS and withdrawing as much fluid as possible. The procedure was repeated 3 times, and the BAL fluid samples were pooled. After centrifugation $(300 \times g$, $5 \mathrm{~min}, 4^{\circ} \mathrm{C}$ ), the supernatants were used for Western blot and lipidomic analysis.

Lipidomic analyses were performed as described previously (Song et al., 2020). Briefly, lipids were extracted from BAL and lung fractions using a modified Bligh and Dyer extraction procedure and dried in a SpeedVac under the on/high mode. Extracted lipids were processed using a highcoverage targeted lipidomics approach. All lipidomic analyses were performed on an Exion LC system coupled with a QTRAP 6500 PLUS system.

\section{RNA extraction and quantitative RT-PCR}

Total RNA was prepared using TRIzol reagent (Ambion, USA) and reverse transcribed with a FastQuant RT Kit (TIANGEN, Beijing, China) according to the manufacturer's protocol. qPCR was performed using SYBR Green in an Agilent Technologies Strata GeneMx3000P system following the manufacturer's instructions. GAPDH was used as an internal control. Primers for amplification are listed in Table $\mathrm{S} 1$ in Supporting Information.

\section{Statistical analysis}

All experiments were repeated at least three times, and all results are presented as the mean \pm standard deviation. For MLI quantification, data were assessed by digital morphometry using the NIS Elements software. A two-tailed Student's $t$-test was used for the comparison of two independent groups. For experiments with more than two groups, oneway ANOVA was performed with appropriate multiple comparisons as described in the figure legends. A nonparametric Kruskal-Wallis test was used for lipidomic analysis. All statistics are representative of biological replicates. *, $P<0.05 ; * *, P<0.01$.

Compliance and ethics The author(s) declare that they have no conflict of interest. This study was performed in accordance with the principles of the Helsinki Declaration of the World Medical Association. All animal experiments were carried out in accordance with the protocols approved by the animal welfare committee of the Institute of Genetics and Developmental Biology, Chinese Academy of Sciences (IGDB-CAS).

Acknowledgements This work was supported by the National Natural Sciences Foundation of China (31730051 and 31601164), the National Key Research and Development Program of China (2018YFA0800900), Natural Science Foundation of Shandong Province, China (ZR2019PH076), and the 
Open Project of Forensic Medicine Key Laboratory of Shanxi Province, China (SFM2019001). We thank Dr. Lin Yang for their input in the electron microscopy analysis experiment. We also wish to thank the American Journal Experts (AJE) editorial team for the contribution in the grammar modification.

\section{References}

Allan, V.J., Thompson, H.M., and McNiven, M.A. (2002). Motoring around the Golgi. Nat Cell Biol 4, E236-E242.

Andreeva, A.V., Kutuzov, M.A., and Voyno-Yasenetskaya, T.A. (2007). Regulation of surfactant secretion in alveolar type II cells. Am J Physiol Lung Cell Mol Physiol 293, L259-L271.

Barinaga-Rementeria Ramirez, I., and Lowe, M. (2009). Golgins and GRASPs: holding the Golgi together. Semin Cell Dev Biol 20, 770779

Baschieri, F., Confalonieri, S., Bertalot, G., Di Fiore, P.P., Dietmaier, W., Leist, M., Crespo, P., Macara, I.G., and Farhan, H. (2014). Spatial control of Cdc42 signalling by a GM130-RasGRF complex regulates polarity and tumorigenesis. Nat Commun 5, 4839.

Baumgart, F., Ospina, O.L., Mingarro, I., Rodríguez-Crespo, I., and PérezGil, J. (2010). Palmitoylation of pulmonary surfactant protein SP-C is critical for its functional cooperation with SP-B to sustain compression/ expansion dynamics in cholesterol-containing surfactant films. Biophys J 99, 3234-3243.

Branchfield, K., Li, R., Lungova, V., Verheyden, J.M., McCulley, D., and Sun, X. (2016). A three-dimensional study of alveologenesis in mouse lung. Dev Biol 409, 429-441.

Brasch, F., Johnen, G., Winn-Brasch, A., Guttentag, S.H., Schmiedl, A., Kapp, N., Suzuki, Y., Müller, K.M., Richter, J., Hawgood, S., et al. (2004). Surfactant protein B in type II pneumocytes and intra-alveolar surfactant forms of human lungs. Am J Respir Cell Mol Biol 30, 449458.

Cabré, E.J., Martínez-Calle, M., Prieto, M., Fedorov, A., Olmeda, B., Loura, L.M.S., and Pérez-Gil, J. (2018). Homo- and heterooligomerization of hydrophobic pulmonary surfactant proteins SP-B and SP-C in surfactant phospholipid membranes. J Biol Chem 293, 9399-9411.

Casals, C., and Cañadas, O. (2012). Role of lipid ordered/disordered phase coexistence in pulmonary surfactant function. Biochim Biophys Acta 1818, 2550-2562.

Crouch, E., and Wright, J.R. (2001). Surfactant proteins A and D and pulmonary host defense. Annu Rev Physiol 63, 521-554.

Curstedt, T., Jörnvall, H., Robertson, B., Bergman, T., and Berggren, P. (1987). Two hydrophobic low-molecular-mass protein fractions of pulmonary surfactant. Characterization and biophysical activity. Eur J Biochem 168, 255-262.

D’Angelo, G., Prencipe, L., Iodice, L., Beznoussenko, G., Savarese, M., Marra, P.F., Di Tullio, G., Martire, G., De Matteis, M.A., and Bonatti, S. (2009). GRASP65 and GRASP55 sequentially promote the transport of $\mathrm{C}$-terminal valine-bearing cargos to and through the Golgi complex. J Biol Chem 284, 34849-34860.

Martínez-Alonso, E., Tomás, M., and Martínez-Menárguez, J.A. (2013). Golgi tubules: their structure, formation and role in intra-Golgi transport. Histochem Cell Biol 140, 327-339.

Froh, D., Gonzales, L.W., and Ballard, P.L. (1993). Secretion of surfactant protein A and phosphatidylcholine from type II cells of human fetal lung. Am J Respir Cell Mol Biol 8, 556-561.

Goerke, J. (1998). Pulmonary surfactant: functions and molecular composition. Biochim Biophys Acta 1408, 79-89.

Goss, V., Hunt, A.N., and Postle, A.D. (2013). Regulation of lung surfactant phospholipid synthesis and metabolism. Biochim Biophys Acta 1831, 448-458.

Griese, M., Kirmeier, H.G., Liebisch, G., Rauch, D., Stückler, F., Schmitz, G., and Zarbock, R. (2015). Surfactant lipidomics in healthy children and childhood interstitial lung disease. PLoS ONE 10, e0117985.
Guttentag, S. (2008). Posttranslational regulation of surfactant protein B expression. Semin Perinatol 32, 367-370.

Hakomori, S.I., and Handa, K. (2015). GM3 and cancer. Glycoconj J 32, 18.

Helenius, A., and Aebi, M. (2001). Intracellular functions of N-linked glycans. Science 291, 2364-2369.

Ikegami, M., and Jobe, A.H. (1998). Surfactant protein metabolism in vivo. Biochim Biophys Acta 1408, 218-225.

Joachim, J., Jefferies, H.B.J., Razi, M., Frith, D., Snijders, A.P., Chakravarty, P., Judith, D., and Tooze, S.A. (2015). Activation of ULK kinase and autophagy by GABARAP trafficking from the centrosome is regulated by WAC and GM130. Mol Cell 60, 899-913.

Johansson, J., and Curstedt, T. (1997). Molecular structures and interactions of pulmonary surfactant components. Eur J Biochem 244, 675-693.

Klionsky, D.J., Abdelmohsen, K., Abe, A., Abedin, M.J., Abeliovich, H., Acevedo Arozena, A., Adachi, H., Adams, C.M., Adams, P.D., Adeli, $\mathrm{K}$., et al. (2016). Guidelines for the use and interpretation of assays for monitoring autophagy (3rd edition). Autophagy 12, 1-222.

Ladinsky, M.S., Mastronarde, D.N., McIntosh, J.R., Howell, K.E., and Staehelin, L.A. (1999). Golgi structure in three dimensions: functional insights from the normal rat kidney cell. J Cell Biol 144, 1135-1149.

Lawson, W.E., Cheng, D.S., Degryse, A.L., Tanjore, H., Polosukhin, V.V., Xu, X.C., Newcomb, D.C., Jones, B.R., Roldan, J., Lane, K.B., et al. (2011). Endoplasmic reticulum stress enhances fibrotic remodeling in the lungs. Proc Natl Acad Sci USA 108, 10562-10567.

Li, Q., Li, Y., Gu, B., Fang, L., Zhou, P., Bao, S., Huang, L., and Dai, X. (2015). Akt phosphorylates Wnt coactivator and chromatin effector Pygo2 at Serine 48 to antagonize its ubiquitin/proteasome-mediated degradation. J Biol Chem 290, 21553-21567.

Li, Q., Jiao, J., Li, H., Wan, H., Zheng, C., Cai, J., and Bao, S. (2018). Histone arginine methylation by Prmt5 is required for lung branching morphogenesis through repression of BMP signaling. J Cell Sci 131, jcs217406.

Liu, C., Mei, M., Li, Q., Roboti, P., Pang, Q., Ying, Z., Gao, F., Lowe, M., and Bao, S. (2017). Loss of the golgin GM130 causes Golgi disruption, Purkinje neuron loss, and ataxia in mice. Proc Natl Acad Sci USA 114, 346-351.

Marra, P., Salvatore, L., Mironov, A., Di Campli, A., Di Tullio, G., Trucco, A., Beznoussenko, G., Mironov, A., and De Matteis, M.A. (2007). The biogenesis of the Golgi ribbon: the roles of membrane input from the ER and of GM130. Mol Biol Cell 18, 1595-1608.

Matsumura, Y., Ban, N., Ueda, K., and Inagaki, N. (2006). Characterization and classification of ATP-binding cassette transporter ABCA3 mutants in fatal surfactant deficiency. J Biol Chem 281, 34503-34514.

Morrisey, E.E., and Hogan, B.L.M. (2010). Preparing for the first breath: genetic and cellular mechanisms in lung development. Dev Cell 18, 823.

Munro, S. (2011). The golgin coiled-coil proteins of the Golgi apparatus. Cold Spring Harbor Perspect Biol 3, a005256.

Nakamura, N., Rabouille, C., Watson, R., Nilsson, T., Hui, N., Slusarewicz, P., Kreis, T.E., and Warren, G. (1995). Characterization of a cis-Golgi Matrix Protein, GM130. J Cell Biol 131, 1715-1726.

Orgeig, S., Hiemstra, P.S., Veldhuizen, E.J.A., Casals, C., Clark, H.W., Haczku, A., Knudsen, L., and Possmayer, F. (2010). Recent advances in alveolar biology: evolution and function of alveolar proteins. Respir Physiol Neurobiol 173, S43-S54.

Paladino, S., Lebreton, S., Tivodar, S., Formiggini, F., Ossato, G., Gratton, E., Tramier, M., Coppey-Moisan, M., and Zurzolo, C. (2014). Golgi sorting regulates organization and activity of GPI proteins at apical membranes. Nat Chem Biol 10, 350-357.

Puthenveedu, M.A., Bachert, C., Puri, S., Lanni, F., and Linstedt, A.D. (2006). GM130 and GRASP65-dependent lateral cisternal fusion allows uniform Golgi-enzyme distribution. Nat Cell Biol 8, 238-248.

Qua Hiansen, J., Keating, E., Aspros, A., Yao, L.J., Bosma, K.J., Yamashita, C.M., Lewis, J.F., and Veldhuizen, R.A.W. (2015). Cholesterol-mediated surfactant dysfunction is mitigated by surfactant protein A. Biochim Biophys Acta 1848, 813-820. 
Roldan, N., Goormaghtigh, E., Pérez-Gil, J., and Garcia-Alvarez, B. (2015). Palmitoylation as a key factor to modulate SP-C-lipid interactions in lung surfactant membrane multilayers. Biochim Biophys Acta 1848, 184-191.

Sauer, B. (1998). Inducible gene targeting in mice using the Cre/lox system. Methods 14, 381-392.

Serrano, A.G., and Pérez-Gil, J. (2006). Protein-lipid interactions and surface activity in the pulmonary surfactant system. Chem Phys Lipids $141,105-118$.

Song, J.W., Lam, S.M., Fan, X., Cao, W.J., Wang, S.Y., Tian, H., Chua, G. H., Zhang, C., Meng, F.P., Xu, Z., et al. (2020). Omics-driven systems interrogation of metabolic dysregulation in COVID-19 pathogenesis. Cell Metab 32, 188-202.e5.

ten Brinke, A., Batenburg, J.J., Gadella, B.M., Haagsman, H.P., Vaandrager, A.B., and van Golde, L.M.G. (2001). The juxtamembrane lysine and arginine residues of surfactant protein $\mathrm{C}$ precursor influence palmitoylation via effects on trafficking. Am J Respir Cell Mol Biol 25, 156-163.

ten Brinke, A., van Golde, L.M.G., and Batenburg, J.J. (2002). Palmitoylation and processing of the lipopeptide surfactant protein C. Biochim Biophys Acta 1583, 253-265.

Tichelaar, J.W., Lu, W., and Whitsett, J.A. (2000). Conditional expression of fibroblast growth factor-7 in the developing and mature lung. J Biol Chem 275, 11858-11864.

van Meer, G. (1998). Lipids of the Golgi membrane. Trends Cell Biol 8, 29-33.

van Meer, G., and Hoetzl, S. (2010). Sphingolipid topology and the dynamic organization and function of membrane proteins. FEBS Lett 584, 1800-1805.
Wang, C., Ye, M., Zhao, Q., Xia, M., Liu, D., He, L., Chen, G., Peng, Y., and Liu, H. (2019). Loss of the Golgi matrix protein 130 cause aberrant IgA1 glycosylation in IgA nephropathy. Am J Nephrol 49, 307-316.

Wang, D., Haviland, D.L., Burns, A.R., Zsigmond, E., and Wetsel, R.A. (2007). A pure population of lung alveolar epithelial type II cells derived from human embryonic stem cells. Proc Natl Acad Sci USA 104, 4449-4454.

Wei, J.H., Zhang, Z.C., Wynn, R.M., and Seemann, J. (2015). GM130 regulates Golgi-derived spindle assembly by activating TPX2 and capturing microtubules. Cell 162, 287-299.

Weide, T., Bayer, M., Köster, M., Siebrasse, J.P., Peters, R., and Barnekow, A. (2001). The Golgi matrix protein GM130: a specific interacting partner of the small GTPase rablb. EMBO Rep 2, 336-341.

Whitsett, J.A., Wert, S.E., and Weaver, T.E. (2015). Diseases of pulmonary surfactant homeostasis. Annu Rev Pathol Mech Dis 10, 371-393.

Wong, M., and Munro, S. (2014). Membrane trafficking. The specificity of vesicle traffic to the Golgi is encoded in the golgin coiled-coil proteins. Science 346, 1256898.

Yeong, F.M. (2013). Multi-step down-regulation of the secretory pathway in mitosis: A fresh perspective on protein trafficking. Bioessays 35, 462-471.

Ying, Z., Mei, M., Zhang, P., Liu, C., He, H., Gao, F., and Bao, S. (2015). Histone arginine methylation by PRMT7 controls germinal center formation via regulating Bcl6 transcription. J Immunol 195, 1538-1547.

Yu, W., Fang, X., Ewald, A., Wong, K., Hunt, C.A., Werb, Z., Matthay, M. A., and Mostov, K. (2007). Formation of cysts by alveolar type II cells in three-dimensional culture reveals a novel mechanism for epithelial morphogenesis. Mol Biol Cell 18, 1693-1700.

\section{SUPPORTING INFORMATION}

The supporting information is available online at https://doi.org/10.1007/s11427-020-1875-x. The supporting materials are published as submitted, without typesetting or editing. The responsibility for scientific accuracy and content remains entirely with the authors. 